\title{
Recent Applications of AC Electrokinetics in Biomolecular Analysis on Microfluidic Devices
}

\author{
Naoki SASAKI \\ Department of Chemical and Biological Sciences, Faculty of Science, Japan Women's University, \\ 2-8-1 Mejirodai, Bunkyo, Tokyo 112-8681, Japan
}

\begin{abstract}
$\mathrm{AC}$ electrokinetics is a generic term that refers to an induced motion of particles and fluids under nonuniform AC electric fields. The AC electric fields are formed by application of AC voltages to microelectrodes, which can be easily integrated into microfluidic devices by standard microfabrication techniques. Moreover, the magnitude of the motion is large enough to control the mass transfer on the devices. These advantages are attractive for biomolecular analysis on the microfluidic devices, in which the characteristics of small space and microfluidics have been mainly employed. In this review, I describe recent applications of AC electrokinetics in biomolecular analysis on microfluidic devices. The applications include fluid pumping and mixing by AC electrokinetic flow, and manipulation of biomolecules such as DNA and proteins by various AC electrokinetic techniques. Future prospects for highly functional biomolecular analysis on microfluidic devices with the aid of $\mathrm{AC}$ electrokinetics are also discussed.
\end{abstract}

(Received August 29, 2011; Accepted September 26, 2011; Published January 10, 2012)

\begin{tabular}{llcc}
1 Introduction & 3 & 4 Biomolecular Manipulation & 5 \\
2 AC Electrokinetics & 4 & $4 \cdot 1$ DNA & \\
3 Fluid Manipulation & 4 & $4 \cdot 2$ Proteins and other biomolecules & \\
$3 \cdot 1$ Fluid pumping & 5 Summary and Outlook & 7 \\
$3 \cdot 2$ Fluid mixing & 6 References & 7 \\
\hline
\end{tabular}

\section{Introduction}

Recent advances in the development of microfluidic devices, which utilize microfluidic channels or microdroplets as workspaces, have led to new applications in bioanalytical chemistry. The devices employ the characteristics of small spaces such as their short diffusion distance, large specific interface area and small heat capacity, and the characteristics of microfluidics such as laminar flow and multiphase flow. ${ }^{1}$ These characteristics promote efficient chemical reactions while offering such practical advantages as reduced sample and reagent volume and high throughput by parallelization. ${ }^{2,3}$ These

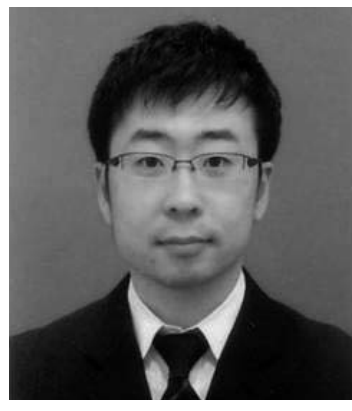

Naoki SASAKI received B. Eng. (2002), M. Eng. (2004) and $\mathrm{PhD}$ in Engineering (2007) degrees (Applied Chemistry) from the University of Tokyo. After working as a Special Postdoctoral Researcher at RIKEN in 2007-2010, he has been serving as an Assistant Professor in the Faculty of Science (Department of Chemical and Biological Sciences) at Japan Women's University. His current research interests include micro- and nanofluidic systems for bioanalysis and bioengineering.

E-mail: sasakina@fc.jwu.ac.jp advantages have made microfluidic devices attractive platforms for disease diagnostics and point-of-care testing, as well as basic scientific tools to study the biological functions of biomolecules. For further development of biomolecular analysis on microfluidic devices, various functions such as pretreatment of samples, mixing and reaction of the samples with reagents, washing, and detection of specific analytes are conducted as required by the operator.

Electrodes have been widely utilized to achieve various functions in biomolecular analysis. They can be easily fabricated on microfluidic devices by standard microfabrication techniques and are operated by applying voltages from an electrical power source. The microfabricated electrodes have been utilized for the electrochemical detection of analytes, ${ }^{4}$ the application of voltages for electrophoretic separations, ${ }^{5}$ and so on. In these applications, the electrodes are energized with DC voltages. On the other hand, application of AC voltages to micrometer-sized electrodes forms strong $\mathrm{AC}$ electric fields, which induce $\mathrm{AC}$ electrokinetic phenomena that do not become evident at the macroscale level.

In this review, I describe recent applications of AC electrokinetics in biomolecular analysis on microfluidic devices. After a brief introduction of AC electrokinetics, I review bioanalytical applications of AC electrokinetics including manipulation of fluids and biomolecules. Finally, I discuss future prospects for highly functional biomolecular analysis on microfluidic devices with the aid of AC electrokinetics. 
(a)

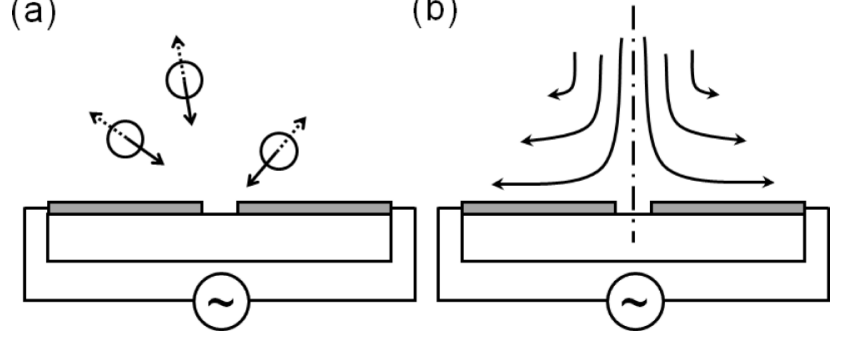

Fig. 1 Schematic illustrations of AC electrokinetics induced by a pair of microelectrodes (gray rectangles) on a planar substrate. (a) DEP of polarizable objects. The solid and dashed arrows indicate the direction of motion of the objects by positive and negative DEP, respectively. (b) AC electrokinetic flow (ACEKF) of fluids. The solid arrows indicate the direction and magnitude of ACEKF.

\section{AC Electrokinetics}

$\mathrm{AC}$ electrokinetics is a generic term that refers to an induced motion of particles and fluids under nonuniform $\mathrm{AC}$ electric fields. ${ }^{6-8}$ The AC electric fields are formed by microelectrodes fabricated on a planar substrate. AC electrokinetics is classified as either dielectrophoresis (DEP) or AC electrokinetic flow (ACEKF). In general, DEP is a motion of polarizable objects (particles, droplets, etc.) under nonuniform electric fields (these can be DC or AC electric fields). ${ }^{9}$ The electric fields induce dipoles on the objects, and the objects are directed toward the higher electric field by positive DEP (p-DEP) or toward the lower electric field by negative DEP (n-DEP), depending on experimental conditions. In the electrode configuration shown in Fig. 1(a), a local maximum in the electric fields is located at the edge of the electrodes. Therefore, the objects are directed toward the edge in the case of p-DEP, or repelled from the edge in the case of n-DEP. ACEKF represents the steady fluid flow induced under nonuniform AC electric fields (Fig. 1(b)). The flow direction is from the center of the electrode gap to the opposite side(s) of the electrodes on the surface, regardless of their instantaneous polarity. ACEKF is further classified as either AC electroosmotic flow (ACEOF) or AC electrothermal flow (ACETF) according to its mechanism. The origin of ACEOF is due to the interaction between the tangential electric field and the charge at the diffuse double layer on the electrodes. ${ }^{10}$ ACEOF is induced particularly in low conductivity solutions and at low frequencies (e.g., $\sigma<8.4 \times 10^{-2} \mathrm{~S} \mathrm{~m}^{-1}$ and $f<$ $100 \mathrm{kHz}) .{ }^{10}$ In contrast, ACETF is induced in high conductivity solutions and at high frequencies. Ions in the solutions are accelerated by the AC electric fields, but they release their kinetic energy as heat (that is, Joule heating) by the collisions with surrounding particles including ions or molecules. Since the electric fields are nonuniform, uneven Joule heating of fluids is produced, which gives rise to nonuniformities in the conductivity and permittivity of the fluids. ${ }^{11}$ These nonuniformities interact with the $\mathrm{AC}$ electric fields to induce ACETF. In addition, other related phenomena such as Brownian motion of particles coexist with these motions. Therefore, it is important to understand the characteristics of each phenomenon in order to apply $\mathrm{AC}$ electrokinetics to bioanalytical processes on microfluidic devices. (a)

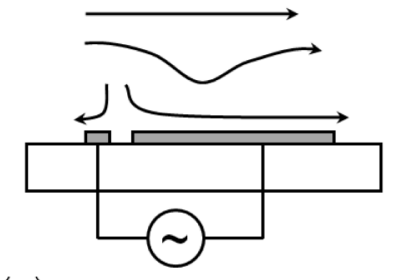

(c)

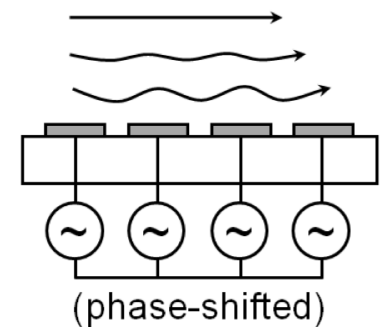

(d)

(b)
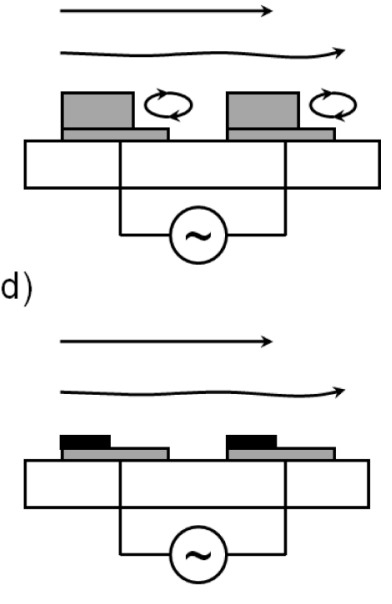

Fig. 2 Schematic illustrations of electrode configurations for fluid pumping by ACEOF. (a) Asymmetric electrodes, (b) three-dimensional stepped electrodes, (c) an electrode array for travelling-wave electroosmosis, (d) electrodes modified partially with dielectrics (black rectangles). The solid arrows indicate the direction and magnitude of ACEOF.

\section{Fluid Manipulation}

Fluid manipulation on microfluidic devices is important for the miniaturization of biomolecular analysis, which often requires pumping and mixing of solutions. In fact, various micropumps and micromixers have been developed. ${ }^{12-14}$ Utilization of ACEOF is one attractive way to manipulate fluids on the devices, because the velocity of ACEOF is sufficiently fast for microscale processes (over $500 \mu \mathrm{m} \mathrm{s}^{-1}$ at several $\mathrm{V}_{\mathrm{p}-\mathrm{p}}$ ). ${ }^{10}$ Additionally, it is easy to control the velocity of ACEOF by the applied voltage and frequency, and to control the flow direction by the electrode configuration. ACETF is also useful since bioanalysis often requires high salt concentration solutions (e.g., physiological saline), in which ACETF becomes evident.

\subsection{Fluid pumping}

ACEOF has been utilized to pump fluids. The direction and magnitude of ACEOF on a pair of coplanar electrodes is symmetrical, as shown around the dashed line of Fig. 1(b). Therefore, the pumping of fluids can be achieved by breaking the symmetry and inducing net flow across the surface of the electrodes. Such an induction of the net flow has been reported using asymmetric pairs of electrodes (Fig. 2(a))..$^{15-27}$ The velocity of the induced flow decreased with increasing the distance from the surface of the electrodes. ${ }^{17-19}$ Such dependence is in contrast with that of pressure-driven flow in a microfluidic channel, which takes maximum value at the center of the channel and falls off to zero with approaching to channel walls. The performance of the pumping depends on the properties of the pumped solution, as well as the parameters of the pump including applied voltages, frequencies, and electrode configurations. For example, a pumping velocity of over $300 \mu \mathrm{m} \mathrm{s}^{-1}$ was achieved with a $10^{-4} \mathrm{M} \mathrm{KCl}$ solution, whereas the velocity decreased to $\sim 60 \mu \mathrm{m} \mathrm{s}^{-1}$ with a $10^{-3} \mathrm{M} \mathrm{KCl}$ solution. ${ }^{20}$ Besides planar electrodes, three-dimensional stepped electrodes have been utilized to achieve pumping (Fig. 2(b)). ${ }^{28-30}$ 
(a)

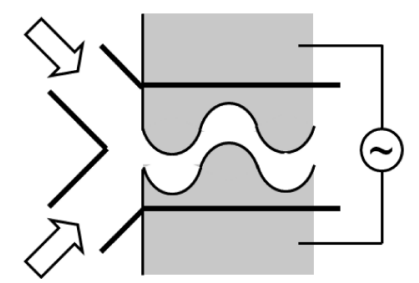

(b)

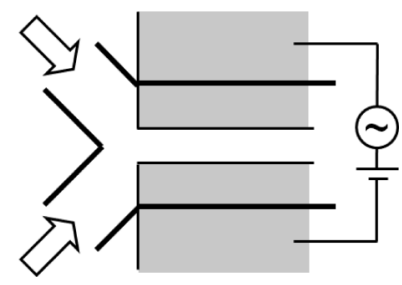

Fig. 3 Schematic illustrations of electrode configurations for fluid mixing by ACEKF. (a) Asymmetric meandering electrodes energized by AC voltages, (b) symmetric electrodes energized by AC voltages with DC bias.

The flow velocity on the electrodes $\left(1300 \mu \mathrm{m} \mathrm{s}^{-1}\right)$ was larger than that on planar electrodes $\left(\sim 300 \mu \mathrm{m} \mathrm{s}^{-1}\right)$. In addition, indirect pumping of buffer solutions at physiological salt concentration was demonstrated using distilled water as working solutions. Such pumping will be beneficial for bioanalytical processes because the performance of the pump is not affected by the properties of the test solutions. Other strategies to pump fluids include applications of phase-shifted AC voltages to an array of the electrodes for traveling-wave electroosmosis (Fig. 2(c)), ${ }^{31,32}$ partial modification of the surface of the electrodes with dielectrics (Fig. 2(d)), ${ }^{33}$ and the use of ACETF. ${ }^{34}$

\subsection{Fluid mixing}

Fluid flow in a microfluidic channel exhibits laminar flow and mixing occurs through diffusion of molecules across the channel. The mixing can be enhanced if ACEKF is induced across the channel and stirs the fluids themselves. A strategy for enhancement is the use of asymmetric electrode configurations to induce net flow of fluids across the channel. Sasaki et al. have developed a micromixer using ACEOF. ${ }^{35}$ A pair of coplanar meandering electrodes was fabricated in the microchannel to induce ACEOF and accelerate mass transfer across the channel (Fig. 3(a)). The mixer was utilized to mix in parallel two streams of water pumped by a syringe pump. The mixing was 20 -fold faster than that of diffusional mixing, and the mixer worked effectively at low Peclet numbers $\left(\mathrm{Pe}<2 \times 10^{3}\right)$ in contrast to typical micromixers ${ }^{36,37}$ that require high Pe. The performance of the mixer was studied under various experimental conditions including applied voltages, frequency, and solution viscosity. ${ }^{38}$ The effects of these conditions were explained by a theoretical model of $\mathrm{ACEOF}^{7}$ and it was possible to estimate the mixing performance under given conditions. The mixer was also applied to fluid mixing by ACETF. ${ }^{39}$ Huang et al. have reported the mixing of two fluids, i.e., high viscosity fluids (90\% glycerol solutions) and low viscosity fluids (aqueous solutions of rhodamine B), as well as the mixing in parallel two streams of water using ACEOF. ${ }^{40}$ Chen et al. also studied fluid mixing in asymmetric electrode configurations. ${ }^{41}$

Another strategy of ACEKF micromixers is the use of DC-biased ACEKF in symmetric electrode configurations as shown in Fig. 3(b). The application of AC voltages with DC bias to the electrodes induces net flow across the channel. $\mathrm{Ng}$ et al. demonstrated such mixing in a Y-shaped microchannel modified with a pair of electrodes. ${ }^{42}$ The mixer was tested at the wide range of conductivity of the solution $\left(\sigma=1-500 \mathrm{mS} \mathrm{m}^{-1}\right)$. The DC-biased AC voltages cause electrolysis of water depending on the experimental conditions, and such effects have been studied. ${ }^{27}$
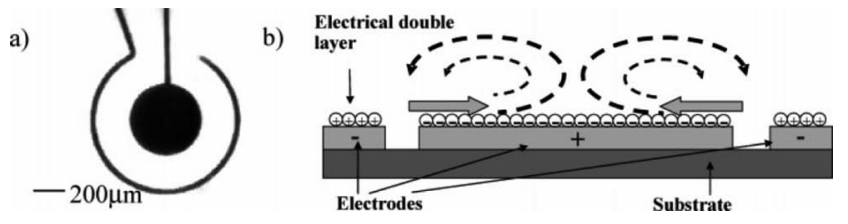

Fig. 4 (a) Electrode design of a DNA concentrator. (b) Schematic (side view) illustrating electrode polarization and induction of ACEOF. Solid arrows represent the AC electroosmotic force and dotted lines indicate the flow pattern. Reproduced with permission from Ref. 45.

\section{Biomolecular Manipulation}

The strategies to manipulate biomolecules by AC electrokinetics on microfluidic devices are as follows: (i) direct manipulation of the biomolecules by DEP, (ii) manipulation of micrometer-sized particles modified with the biomolecules by DEP, and (iii) manipulation of fluids that contain the biomolecules by ACEKF. In general, DEP is effective for the manipulation of micrometer-sized particles, large DNA molecules ${ }^{43}$ and biopolymers, ${ }^{44}$ but not for small molecules. For example, assuming that the gap between the electrodes shown in Fig. 1(a) is $25 \mu \mathrm{m}$ and that the voltage applied to the electrodes is $5 \mathrm{~V}_{\mathrm{p}-\mathrm{p}}$, one find that the radius of the objects manipulated by DEP should be more than $0.2 \mu \mathrm{m} .^{7}$ In addition, the magnitude of the DEP is inversely proportional to the cube of the distance from the interelectrode gap, ${ }^{7}$ which means DEP is only effective near the gap. In contrast, ACEKF can manipulate the biomolecules regardless of their size, through the manipulation of the fluids that contain the molecules. Moreover, the magnitude of ACEKF is inversely proportional to the distance from the interelectrode gap, which means ACEKF is effective at a longer range compared to DEP.

\subsection{DNA}

Enrichment of DNA molecules to a specific location on microfluidic devices has been reported. Wong et al. have reported a DNA concentrator that can enrich $\lambda$-phage DNA and 20-base single-stranded (ss) DNA on a circular microelectrode surrounded by a ring electrode (Fig. 4). ${ }^{45}$ The DNA molecules were enriched by the simultaneous application of AC and DC bias voltages to the electrodes. Convection of fluids by ACEOF and accumulation of the DNA molecules to the electrodes by electrophoretic force were suggested. Lei et al. studied the effects of the applied voltages on the enrichment in detail. ${ }^{46}$ When the DC bias voltage was above $1 \mathrm{~V}$, the ssDNA molecules were concentrated not on the circular electrode, but in the gap between the two electrodes. The authors suggested that ions in the solutions accumulated on the circular electrode and that the ssDNA molecules were repelled from the electrode. Yokokawa et al. reported the detailed study of the enrichment on transparent indium tin oxide electrodes. ${ }^{47}$ They measured the ACEOF velocity on the electrodes, and the dependence of the velocity on frequency agreed with theoretical estimations, although the magnitude of the velocity was different. In addition, three-dimensional accumulation of the DNA molecules was observed. Bown and Meinhart measured the fluid velocity profile around the electrodes by a two-color micro-particle image velocimetry technique. ${ }^{48}$ The obtained velocity profile agreed with simulated results except for a discrepancy in the magnitude of the velocity. In the simulation, the incompressible Stokes equations and the mass continuity equation were solved 


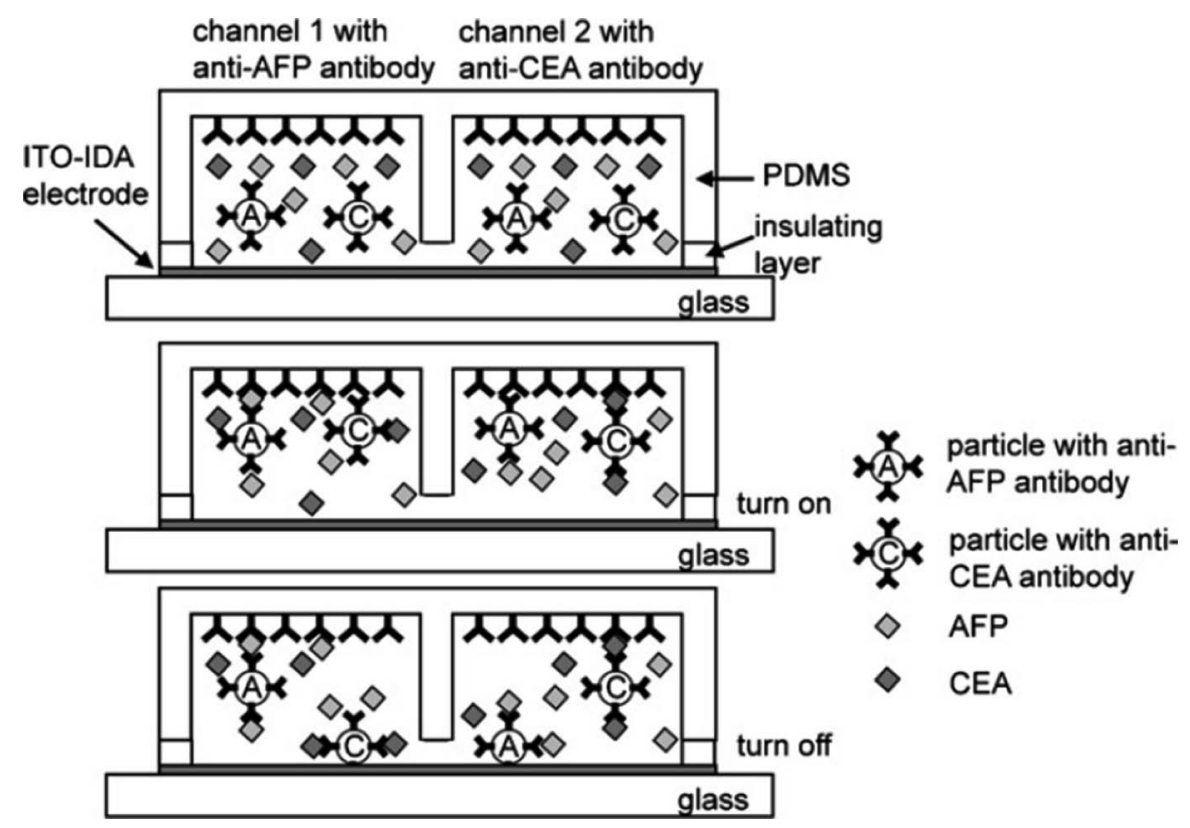

Fig. 5 Schematic illustrations of a sandwich immunoassay based on n-DEP particle manipulation. Reproduced with permission from Ref. 52.

for fluid motion, and ACEOF on the electrodes was included as a boundary condition. The authors suggested that the potential drop across the electric double layer, which is an important parameter of ACEOF, might not be estimated accurately. Du et al. also reported concentration enrichment of T4 DNA on microelectrodes under AC electric fields. ${ }^{49,50}$ These techniques are expected to be utilized as an important operation, e.g., preconcentration of analytes prior to electrophoretic separation, in microfluidic DNA analysis.

\subsection{Proteins and other biomolecules}

Yasukawa et al. utilized n-DEP trapping of antibody-modified microparticles in a microchannel to perform a sandwich immunoassay. ${ }^{51}$ Immunoreactions took place on the surface of the trapped particles under continuous flow of test solutions, and therefore unreacted antigens and secondary antibodies were automatically separated from the particles. Mouse immunoglobulin $\mathrm{G}$ ( $\mathrm{IgG}$ ) was detected in the range 0.01 $10 \mu \mathrm{g} \mathrm{mL}^{-1}$. Lee et al. have reported sandwich immunoassay of tumor markers ( $\alpha$-fetoprotein and carcinoembryonic antigen) by n-DEP manipulation of antibody-modified microparticles..$^{52}$ The particles were pushed toward the channel surface where primary antibodies were adsorbed, and attached irreversibly when the target antigens were in the solution (Fig. 5). The antigens were successfully detected in the range 0.1 $100 \mathrm{ng} \mathrm{mL}^{-1}$, which covers the concentration required for medical diagnostics.

Besides microparticles, microdroplets can be manipulated by DEP. Sasaki et al. have reported a combination of cloud point extraction, which has been reviewed by Tani et al.,$^{53}$ and n-DEP of microdroplets to achieve enrichment of membrane-associated biomolecules. ${ }^{54}$ A test solution containing the molecules and nonionic surfactants was continuously introduced into an electrode-integrated microfluidic channel. Application of AC voltages to the electrodes raises the temperature of the solution above its cloud point by Joule heating, which leads to the generation of microdroplets of a surfactant-rich phase by micellar aggregation. As a result, the microdroplets were

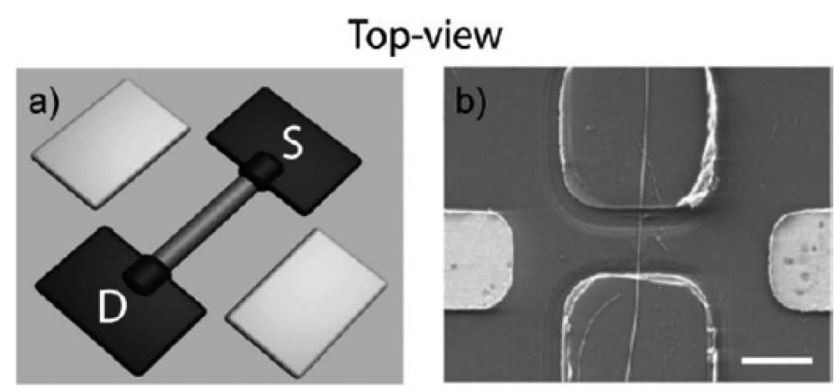

Side-view

c)
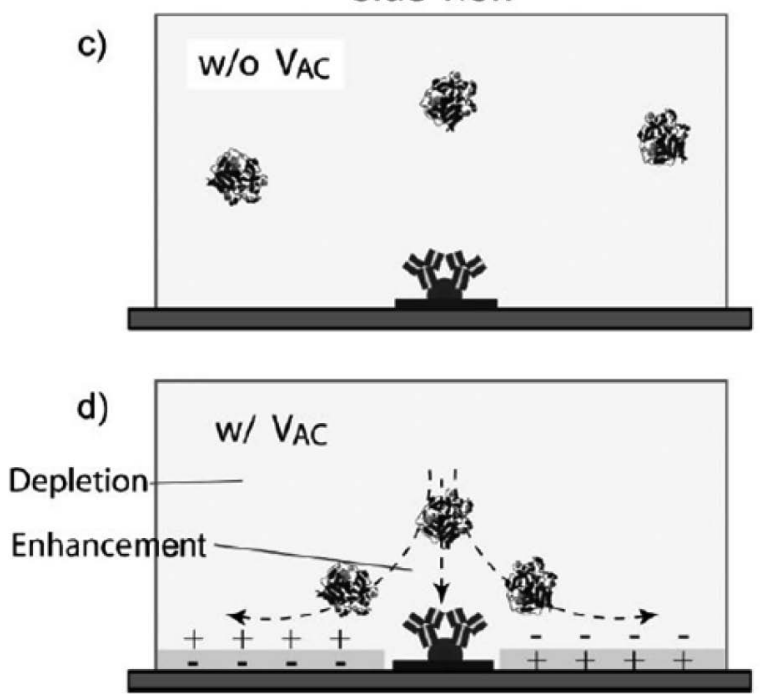

Fig. 6 (a) Schematic image of the microfabricated device integrated with microelectrodes and nanowire field-effect transistors. The nanowire is contacted with two electrodes, source (S) and drain (D), to measure conductance. Another pair of microelectrodes surrounds the wire. (b) SEM image of the device. Scale bar is $2 \mu \mathrm{m}$. (c) - (d) Schematic illustrations of protein binding to the nanowire modified with antibodies in a microfluidic channel. (c) Diffusional binding, and (d) enhanced binding under nonuniform AC electric fields. Reproduced with permission from Ref. 58. 
Table 1 Improved detection of biomolecules with AC electrokinetics ${ }^{\mathrm{a}}$

\begin{tabular}{|c|c|c|c|c|c|c|c|}
\hline Analyte & Receptor & Detection & [Analyte]/M & Solution & Time/min & Improvement & Ref. \\
\hline SA-PE & Biotin & FL & $3.3 \times 10^{-8 b}$ & $0.1 \mathrm{M} \mathrm{PB}$ & 5 & $7.8 \pm 1.7^{\mathrm{f}}$ & 55 \\
\hline FITC-IgG & Anti-IgG & FL & $5.9 \times 10^{-8 c}$ & $10^{-5} \mathrm{M} \mathrm{KCl}^{\mathrm{d}}$ & 15 & $6.7^{\mathrm{f}}$ & 56 \\
\hline FITC-IgG & $\mathrm{Au}$ & QCM & $5.9 \times 10^{-8 c}$ & $10^{-5} \mathrm{M} \mathrm{KCl}^{\mathrm{d}}$ & 15 & $5.6^{\mathrm{f}}$ & 57 \\
\hline PSA & Anti-PSA & FET & $1.0 \times 10^{-17}$ & $10^{-5} \mathrm{M} \mathrm{PB}$ & $-\mathrm{e}$ & $10^{4 \mathrm{~g}}$ & 58 \\
\hline СТB & Ganglioside & FET & $1.8 \times 10^{-17}$ & $10^{-5} \mathrm{M}$ PB & $-\mathrm{e}$ & $10^{4 \mathrm{~g}}$ & 58 \\
\hline
\end{tabular}

a. FL, fluorescence; PB, phosphate buffer. b. Calculated from the concentration $\left(10 \mu \mathrm{g} \mathrm{mL} \mathrm{m}^{-1}\right)$ and molecular weight $(300000)$ of SA-PE. c. Calculated from the concentration $\left(10 \mu \mathrm{g} \mathrm{mL}^{-1}\right)$ and molecular weight (170000) of FITC-IgG. d. Estimated from the conductivity of the solution $\left(2 \times 10^{-4} \mathrm{~S} \mathrm{~m}^{-1}\right)$. The variation of the conductivity of an aqueous $\mathrm{KCl}$ solution with concentration is shown in Ref. 8 . e. AC voltages are applied throughout the measurements. f. Signal enhancement. g. Increase in sensitivity.

trapped above the electrodes by n-DEP forces, and enrichment of the target molecules was achieved. Fluorescently-labeled lipids were used as model molecules, and fluorescent intensities of the trapped lipids increased by a factor of $\sim 40$ in $1 \mathrm{~min}$.

Manipulation of biomolecules by ACEKF has also been studied. Sigurdson et al. proposed the stirring of fluids by ACETF to enhance the transfer of analytes to a surface of an electrode-integrated microfluidic channel. ${ }^{11} \quad$ Numerical simulations of fluid dynamics and consequent distribution of analytes in the channel revealed that the stirring is effective when the reaction is diffusion-limited. Such an enhancement was demonstrated later by Feldman et al..$^{55}$ A pair of coplanar electrodes was utilized to enhance the reaction of streptavidin R-phycoerythrin (SA-PE) with biotin immobilized on the channel surface. Binding of the SA-PE was enhanced by a factor of $\sim 9$ in $15 \mathrm{~min}$. Hart et al. studied the enhancement of immunoreactions by the stirring of fluids by ACEOF. ${ }^{56}$ Numerical simulations were utilized to optimize electrode configurations and achieve efficient enhancement. The amounts of fluorescein isothiocyanate-IgG (FITC-IgG) that were bound to immobilized IgG were enhanced after the application of AC voltages. The fluorescent signals from FITC-IgG increased by a factor of 6.7 at the edge of the electrodes. In contrast, signals increased only by a factor of 1.9 at the center of the electrodes. Therefore, p-DEP trapping of FITC-IgG at the edge of the electrodes was also suggested.

Recently, combinations of AC electrokinetics with various analytical techniques have been reported. Hart et al. reported an improved detection of proteins on a quartz crystal microbalance (QCM) with the aid of AC electrokinetics. ${ }^{57} \mathrm{~A}$ side of the QCM was modified with interdigitated electrodes to induce ACEOF and to accelerate the transfer of the proteins to the side. FITC-IgG was used as a model protein, and the mass adsorbed on the QCM increased by a factor of 5.6. Gong reported label-free and sensitive detection of prostate specific antigen (PSA) on microfabricated devices integrated with microelectrodes and nanowire field-effect transistors (FET) ${ }^{58}$ The device is composed of a pair of microelectrodes connected with a piece of silicon nanowire, and another pair of microelectrodes that surrounds the wire (Figs. 6(a) and 6(b)). The former electrodes act as a sensor for the PSA, while the latter electrodes induce ACEOF and accelerate the transfer of the PSA to the surface of the sensor (Figs. 6(c) and 6(d)). The sensitivity of the sensor increased by a factor of $10^{4}$ with AC voltage application, which allowed detection of 10 aM PSA. Versatility of the method was demonstrated by the detection of another analyte, cholera toxin subunit B (CTB) with ganglioside.

To present an overview of the effect of AC electrokinetics on biomolecular detection, I summarize typical experimental conditions and results shown in the aforementioned papers in
Table 1. The conditions include analytes, receptors that capture the analytes on a specific location, detection techniques, the concentration of analytes, solution composition in measurements, and the time required for voltage application prior to the measurements. Improvements in each experiment are indicated in the form of signal enhancement or an increase in sensitivity. It is clearly shown that $\mathrm{AC}$ electrokinetics can contribute to signal enhancement in a short analysis time. Moreover, ultrasensitive detection of analytes can be achieved using FET. Although these experiments were mainly conducted in low salt concentration conditions where the effect of ACEOF is evident, further application in high salt concentration conditions will expand the applicability of the techniques. Applications to real samples which often contain various types of molecules should also be investigated.

\section{Summary and Outlook}

I have reviewed recent applications of $\mathrm{AC}$ electrokinetics for biomolecular analysis on microfluidic devices. AC electrokinetics was successfully utilized to manipulate fluids, particles, and biomolecules. Pumping and mixing of fluids by ACEKF was demonstrated using several electrode configurations and operations. In addition to the fluids, biomolecules were successfully manipulated by AC electrokinetics through the manipulation of particles and fluids. The manipulation led to the enrichment of biomolecules on a specific location on the microfluidic devices, which resulted in enhanced detection of analytes in a short time. These results show some prospects for future biomolecular analysis on microfluidic devices. In particular, the integration of multiple operations with the aid of AC electrokinetics will lead to the development of highly functional microdevices for biomolecular analysis. Although the existing AC electrokinetic operations mainly employ coplanar electrodes that have a micrometer-sized interelectrode gap, the use of nano-gap electrodes will result in the formation of stronger AC electric fields and thus more effective operations. The challenges detailed above will open up new possibilities in the fields of biomolecular analysis and AC electrokinetics.

\section{References}

1. T. Kitamori, M. Tokeshi, A. Hibara, and K. Sato, Anal. Chem., 2004, 76, 52A.

2. T. G. Henares, M. Takaishi, N. Yoshida, S. Terabe, F. Mizutani, R. Sekizawa, and H. Hisamoto, Anal. Chem., 2006, 79, 908.

3. R. Gomez-Sjoberg, A. A. Leyrat, D. M. Pirone, C. S. Chen, 
and S. R. Quake, Anal. Chem., 2007, 79, 8557.

4. K. Ueno, H.-B. Kim, and N. Kitamura, Anal. Chem., 2003, 75, 2086.

5. A. Inoue, T. Ito, K. Makino, K. Hosokawa, and M. Maeda, Anal. Chem., 2007, 79, 2168.

6. A. Ramos, H. Morgan, N. G. Green, and A. Castellanos, J. Phys. D: Appl. Phys., 1998, 31, 2338.

7. A. Castellanos, A. Ramos, A. Gonzalez, N. G. Green, and H. Morgan, J. Phys. D: Appl. Phys., 2003, 36, 2584.

8. H. Morgan and N. G. Green, "AC Electrokinetics: Colloids and Nanoparticles", 2003, Research Studies Press Ltd., Baldock, Hertfoldshire, England.

9. H. A. Pohl, "Dielectrophoresis", 1978, Cambridge University Press, Cambridge, London, New York, Melbourne.

10. N. G. Green, A. Ramos, A. Gonzalez, H. Morgan, and A. Castellanos, Phys. Rev. E, 2000, 61, 4011.

11. M. Sigurdson, D. Z. Wang, and C. D. Meinhart, Lab Chip, 2005, 5, 1366.

12. B. Iverson and S. Garimella, Microfluid. Nanofluid., 2008 , $5,145$.

13. A. Nisar, N. Afzulpurkar, B. Mahaisavariya, and A. Tuantranont, Sens. Actuators, B, 2008, 130, 917.

14. C.-C. Chang and R.-J. Yang, Microfluid. Nanofluid., 2007, $3,501$.

15. A. Ajdari, Phys. Rev. E, 2000, 61, R45.

16. V. Studer, A. Pépin, Y. Chen, and A. Ajdari, Microelectron. Eng., 2002, $61-62,915$.

17. A. B. D. Brown, C. G. Smith, and A. R. Rennie, Phys. Rev. E, 2001, 63, 016305.

18. M. Mpholo, C. G. Smith, and A. B. D. Brown, Sens. Actuators, B, 2003, 92, 262.

19. A. Ramos, A. Gonzalez, A. Castellanos, N. G. Green, and H. Morgan, Phys. Rev. E, 2003, 67, 056302.

20. V. Studer, A. Pepin, Y. Chen, and A. Ajdari, Analyst, 2004, 129, 944.

21. S. Debesset, C. J. Hayden, C. Dalton, J. C. T. Eijkel, and A. Manz, Lab Chip, 2004, 4, 396.

22. D. Lastochkin, R. Zhou, P. Wang, Y. Ben, and H.-C. Chang, J. Appl. Phys., 2004, 96, 1730.

23. L. H. Olesen, H. Bruus, and A. Ajdari, Phys. Rev. E, 2006, $73,056313$.

24. M. M. Gregersen, L. H. Olesen, A. Brask, M. F. Hansen, and H. Bruus, Phys. Rev. E, 2007, 76, 056305.

25. N. Loucaides, A. Ramos, and G. Georghiou, Microfluid. Nanofluid., 2007, 3, 709.

26. S.-H. Huang, H.-J. Hsueh, and K.-Y. Hung, Microfluid. Nanofluid., 2010, 8, 187.

27. W. Y. Ng, Y. C. Lam, and I. Rodriguez, Biomicrofluidics, 2009, 3, 022405.

28. M. Z. Bazant and Y. Ben, Lab Chip, 2006, 6, 1455.

29. J. P. Urbanski, T. Thorsen, J. A. Levitan, and M. Z. Bazant, Appl. Phys. Lett., 2006, 89, 143508.

30. C.-C. Huang, M. Z. Bazant, and T. Thorsen, Lab Chip, 2010, 10,80 .

31. A. Ramos, H. Morgan, N. G. Green, A. González, and A.
Castellanos, J. Appl. Phys., 2005, 97, 084906.

32. B. P. Cahill, L. J. Heyderman, J. Gobrecht, and A. Stemmer, Sens. Actuators, B, 2005, 110, 157.

33. C.-T. Kuo and C.-H. Liu, Lab Chip, 2008, 8, 725.

34. J. Wu, M. Lian, and K. Yang, Appl. Phys. Lett., 2007, 90.

35. N. Sasaki, T. Kitamori, and H.-B. Kim, Lab Chip, 2006, 6, 550.

36. A. D. Stroock, S. K. W. Dertinger, A. Ajdari, I. Mezic, H. A. Stone, and G. M. Whitesides, Science, 2002, 295, 647.

37. R. H. Liu, M. A. Stremler, K. V. Sharp, M. G. Olsen, J. G. Santiago, R. J. Adrian, H. Aref, and D. J. Beebe, J. Microelectromech. Syst., 2000, 9, 190.

38. N. Sasaki, T. Kitamori, and H.-B. Kim, Anal. Sci., 2010, 26,815 .

39. N. Sasaki, T. Kitamori, and H.-B. Kim, in "Micro Total Analysis Systems 2007”, ed. J.-L. Viovy, P. Tabeling, S. Descroix, and L. Malaquin, 2007, Paris, 916.

40. S.-H. Huang, S.-K. Wang, H. S. Khoo, and F.-G. Tseng, Sens. Actuators, B, 2007, 125, 326.

41. J.-K. Chen, C.-N. Weng, and R.-J. Yang, Lab Chip, 2009, 9, 1267.

42. W. Y. Ng, S. Goh, Y. C. Lam, C. Yang, and I. Rodriguez, Lab Chip, 2009, 9, 802.

43. M. Washizu and O. Kurosawa, IEEE Trans. Ind. Appl., 1990, 26, 1165.

44. M. Washizu, S. Suzuki, O. Kurosawa, T. Nishizaka, and T. Shinohara, IEEE Trans. Ind. Appl., 1994, 30, 835.

45. P. K. Wong, C.-Y. Chen, T.-H. Wang, and C.-M. Ho, Anal. Chem., 2004, 76, 6908.

46. K. F. Lei, H. Cheng, K. Y. Choy, and L. M. C. Chow, Sens. Actuators, A, 2009, 156, 381.

47. R. Yokokawa, Y. Manta, M. Namura, Y. Takizawa, N. C. H. Le, and S. Sugiyama, Sens. Actuators, B, 2010, 143, 769.

48. M. Bown and C. Meinhart, Microfluid. Nanofluid., 2006, 2, 513.

49. J.-R. Du, Y.-J. Juang, J.-T. Wu, and H.-H. Wei, Biomicrofluidics, 2008, 2, 044103.

50. J.-R. Du and H.-H. Wei, Biomicrofluidics, 2010, 4, 034108.

51. T. Yasukawa, M. Suzuki, T. Sekiya, H. Shiku, and T. Matsue, Biosens. Bioelectron., 2007, 22, 2730.

52. H. J. Lee, S. H. Lee, T. Yasukawa, J. Ramón-Azcón, F. Mizutani, K. Ino, H. Shiku, and T. Matsue, Talanta, 2010, $81,657$.

53. H. Tani, T. Kamidate, and H. Watanabe, Anal. Sci., 1998, 14, 875.

54. N. Sasaki, K. Hosokawa, and M. Maeda, Lab Chip, 2009, 9, 1168.

55. H. C. Feldman, M. Sigurdson, and C. D. Meinhart, Lab Chip, 2007, 7, 1553.

56. R. Hart, R. Lec, and H. M. Noh, Sens. Actuators, B, 2010, 147, 366.

57. R. Hart, E. Ergezen, R. Lec, and H. M. Noh, Biosens. Bioelectron., 2011, 26, 3391.

58. J.-R. Gong, Small, 2010, 6, 967. 